\title{
Modified Zakharov equations for plasmas with a quantum correction
}

\author{
L. G. Garcia, F. Haas, L. P. L. de Oliveira and J. Goedert* \\ Universidade do Vale do Rio dos Sinos - UNISINOS \\ Aa. Unisinos, 950 \\ 93022-000 São Leopoldo, RS, Brazil
}

\begin{abstract}
Quantum Zakharov equations are obtained to describe the nonlinear interaction between quantum Langmuir waves and quantum ion-acoustic waves. These quantum Zakharov equations are applied to two model cases, namely the four-wave interaction and the decay instability. In the case of the four-wave instability, sufficiently large quantum effects tend to suppress the instability. For the decay instability, the quantum Zakharov equations lead to results similar to those of the classical decay instability except for quantum correction terms in the dispersion relations. Some considerations regarding the nonlinear aspects of the quantum Zakharov equations are also offered.
\end{abstract}

PACS numbers: 52.35.-g, 52.35.Dm, 52.35.Sb

$\bar{*}$ \{garcia, ferhaas, luna, goedert\}@exatas.unisinos.br 


\section{INTRODUCTION}

The importance of quantum effects in ultra-small electronic devices, $\frac{1}{2}$ in dense astrophysical plasma systems ${ }^{2}$ and in laser plasmas ${ }^{3}$ have produced an increasing interest on the investigation of the quantum counterpart of some of the classical plasma physics phenomena. For instance, quantum plasma echoes $\underset{,}{-4}$ the expansion of a quantum electron gas into vacuum,,$\frac{5}{-}$ the quantum two and three stream instabilities ${ }^{6}$ and the quantum Landau damping ${ }^{7}$ have been the subject of recent investigations. Also, quantum methods like the Wigner-Moyal transform have been used in the treatment of the Landau damping of classical partially incoherent Langmuir waves. $\frac{8}{-}$ Quantum models like the Schrödinger-Poisson system have also been used, through the correspondence principle, for the numerical simulation of the Vlasov-Poisson system. ${ }^{9}$

In this context, a mathematical formulation, based on the quantum hydrodynamical model for charged particle systems, $\stackrel{10.11}{~ w a s ~ i n t r o d u c e d ~ t o ~ s t u d y ~ t h e ~ q u a n t u m ~ v e r s i o n ~ o f ~}$ the low frequency ion-acoustic waves. ${ }^{12}$ In micro-electronics, the quantum hydrodynamical model describes $\frac{13}{3}$ negative differential resistance associated to resonant tunnelling diodes. It can also model ultra-small high-electron-mobility transistors. ${ }^{14}$ The quantum hydrodynamical model for charged particle systems was also successfully used for the description of quantum dissipation,,$\frac{15}{5}$ under the same closure hypothesis as that adopted by Manfredi and Haas. ${ }^{11}$ In the case of quantum ion-acoustic waves, ${ }^{12}$ several features of pure quantum origin were observed for the linear, weakly nonlinear and fully nonlinear waves. The linear quantum ion-acoustic waves are described by a dispersion relation which tends to the classical dispersion relation as quantum effects goes to zero, in accordance with the correspondence principle. The weakly nonlinear quantum ion-acoustic waves are described by a modified Korteweg-de Vries equation depending on a scaled $\hbar$ parameter. Finally, the fully nonlin-

ear quantum ion-acoustic waves can have a coherent, periodic pattern, not present in the classical case. This points to the intrinsically more reversible features of quantum plasmas, as seen for instance in quantum echoes ${ }^{4}$ and coherent patterns in the quantum two stream instabilities $\underline{6}$.

The purpose of the present paper is to continue this investigation by studying the nonlinear coupling between the quantum ion-acoustic waves and the quantum Langmuir waves. At the classical level, a set of coupled nonlinear wave equations describing the interaction 
between high frequency Langmuir waves and low frequency ion-acoustic waves was first derived by Zakharov ${ }^{16}$ Since then, this system have been the subject of a large number of studies. $\frac{17}{}$ In one-dimension, the Zakharov equations can be written (in normalized units) as

$$
\begin{aligned}
& i \frac{\partial E}{\partial t}+\frac{\partial^{2} E}{\partial x^{2}}=n E, \\
& \frac{\partial^{2} n}{\partial t^{2}}-\frac{\partial^{2} n}{\partial x^{2}}=\frac{\partial^{2}|E|^{2}}{\partial x^{2}},
\end{aligned}
$$

where $E$ is the envelope of the high frequency electric field and $n$ is the plasma density measured from its equilibrium value. The system (102) can be derived from a hydrodynamic description of the plasma $\frac{17.18}{}$ by distinguishing two different time scales, the slow time scale of the ions and the fast time scale of the electrons. The low mobility of the ions as compared to that of the electrons justifies this kind of treatment. Since the Landau damping of the Langmuir waves is neglected in the fluid description, the model (10/2) is restricted by the condition $k \ll k_{D}$, where $k$ is the wavenumber and $k_{D}$ is the Debye wavenumber. Also, a weak turbulence condition is to be satisfied. $\underline{\underline{17}}$

In this paper, modified Zakharov equations are obtained by use of a quantum fluid approach. Specifically, we assume a two species, one-dimensional quantum plasma in the electrostatic approximation. Pressure effects are neglected for the ions whereas the electrons are described by an isothermal equation of state. Contrary to the quantum degenerate case $\frac{12}{\underline{2}}$ the present model is more suitable to investigate the classical limit $\hbar \rightarrow 0$.We do not include quantum statistical effects in the present investigation, and therefore, only quantum diffraction effects, responsible e.g. for tunnelling, are taken into account.

The paper is organized as follows. In Section II we write the quantum hydrodynamic model for a two-species plasma and derive the Langmuir mode for quantum plasmas. In Section III, we obtain the quantum Zakharov system through a procedure similar to the classical one where a two-time scale formalism is used. In Section IV we study the influence of quantum effects in two relevant parametric instabilities: the decay instability and the four-wave instability. Section VI is devoted to a preliminary discussion of the nonlinear aspects of the problem and some of the remaining open questions. Section V is reserved to the conclusions. 


\section{QUANTUM LANGMUIR WAVES}

Before considering the nonlinear coupling between ion-acoustic and Langmuir waves, we examine the linear stability analysis of the Langmuir waves in the quantum regime. ${ }^{19}$ For this purpose we consider a one-dimensional quantum system, composed of electrons and singly charged ions. The quantum hydrodynamic equations in this case become ${ }^{12}$

$$
\begin{aligned}
& \frac{\partial n_{e}}{\partial t}+\frac{\partial\left(n_{e} u_{e}\right)}{\partial x}=0 \\
& \frac{\partial n_{i}}{\partial t}+\frac{\partial\left(n_{i} u_{i}\right)}{\partial x}=0 \\
& \frac{\partial u_{e}}{\partial t}+u_{e} \frac{\partial u_{e}}{\partial x}=-\frac{e}{m_{e}} E-\frac{1}{m_{e} n_{e}} \frac{\partial P_{e}}{\partial x}+\frac{\hbar^{2}}{2 m_{e}^{2}} \frac{\partial}{\partial x}\left(\frac{\partial^{2} \sqrt{n_{e}} / \partial x^{2}}{\sqrt{n_{e}}}\right), \\
& \frac{\partial u_{i}}{\partial t}+u_{i} \frac{\partial u_{i}}{\partial x}=\frac{e}{m_{i}} E \\
& \frac{\partial E}{\partial x}=\frac{e}{\varepsilon_{0}}\left(n_{i}-n_{e}\right)
\end{aligned}
$$

where $E$ is the electric field, $P_{e}$ is the electron pressure, and $n_{e}, n_{i}, u_{e}, u_{i}, m_{e}$ and $m_{i}$ represent the density, fluid velocity and mass of electrons $(e)$ and ions $(i)$, respectively. In addition, $\varepsilon_{0}$ and $\hbar$ are the vacuum dielectric and the scaled Planck's constants. Since we are interested in high frequency waves, the ion density $n_{i}$ can be assumed constant, at this stage. The pressure $P_{e}$ is obtained from an equation of state for the electrons, which basically depends on the thermodynamic properties of the system. In the present investigation, we consider the isothermal equation of state $P_{e}=\kappa_{B} n_{e} T_{e}$, where $T_{e}$ is the electrons' temperature and $\kappa_{B}$ is the Boltzmann's constant. In view of their large mass, ions are treated classically. Also, in a first approximation, we consider cold, zero temperature ions. The Bohm potential term proportional to $\hbar^{2}$ in (5) is responsible for negative differential resistance in semiconductor devices ${ }^{13}$ and is associated to tunnelling.

Linearization of the electron equations (3), (5) and (17) around the homogeneous equilibrium $n_{e}=n_{i}=n_{0}, u_{e}=0$ and $E=0$ produces the following dispersion relation:

$$
\omega^{2}=\omega_{e}^{2}+v_{e}^{2} k^{2}+\frac{\hbar^{2}}{4 m_{e}^{2}} k^{4} .
$$

In Eq. (8), $\omega$ is the wave frequency, $k$ is the wavenumber, $\omega_{e}=\left(n_{0} e^{2} / m_{e} \varepsilon_{0}\right)^{1 / 2}$ is the electron plasma frequency and $v_{e}=\left(\kappa_{B} T_{e} / m_{e}\right)^{1 / 2}$ is the electron thermal velocity. Notice that both classical and quantum modes can be obtained from Eq. (8) . In fact, the classical limit $\hbar \rightarrow 0$ 
gives the classical Langmuir wave dispersion relation. 18 According to Eq. (8), the frequency $\omega$ is always real, and instability (or damping) of this wave cannot be observed. The main purpose of this paper is to obtain a model describing the exchange of energy between the quantum Langmuir modes shown above and the recently found quantum ion-acoustic plasma modes. 12

\section{QUANTUM ZAKHAROV EQUATIONS}

In order to obtain the set of equations describing the nonlinear interaction between Langmuir waves and ion-acoustic waves, in the quantum regime, we follow the derivation originally made by Zakharov ${ }^{16}$ A general discussion of the validity of the Zakharov equations can be found in the review paper by Thornhill and ter Haar. 17

We first separate all fluid variables into high frequency (subscript $h$ ) and low frequency (subscript $l$ ) components,

$$
\begin{aligned}
& n_{e}(x, t)=n_{0}+n_{l}(x, t)+n_{h}(x, t), \\
& n_{i}(x, t)=n_{0}+n_{l}(x, t), \\
& u_{e}(x, t)=u_{l}(x, t)+u_{h}(x, t), \\
& u_{i}(x, t)=u_{l}(x, t), \\
& E(x, t)=E_{l}(x, t)+E_{h}(x, t) .
\end{aligned}
$$

Notice that the high frequency portions of the ion quantities [Eqs. (10) and (12)] were ignored due to the large ion mass. Also, from the very beginning we assume that departures from the quasi-neutral regime $\left(n_{i} \approx n_{e}\right.$ and $\left.u_{i} \approx u_{e}\right)$ are provided only by the high frequency components of the electrons motion. The high frequency term of the electric field can also be written as

$$
E_{h}(x, t)=\frac{1}{2} \tilde{E}(x, t) e^{-i \omega_{e} t}+\text { c.c. }
$$

where $\tilde{E}(x, t)$ is the slowly varying envelope of the high frequency term and c.c. refer to complex conjugate. Using the high frequency components of Eqs. (3) (7), we obtain, by the same procedure used in the classical case,$\frac{17}{17}$

$$
i \frac{\partial \tilde{E}}{\partial t}+\frac{1}{2} \frac{v_{e}^{2}}{\omega_{e}} \frac{\partial^{2} \tilde{E}}{\partial x^{2}}-\frac{\hbar^{2}}{8 m_{e}^{2} \omega_{e}} \frac{\partial^{4} \tilde{E}}{\partial x^{4}}=\frac{\omega_{e}}{2} \frac{n_{l}}{n_{0}} \tilde{E}
$$


where the term $\left|\partial_{t}^{2} \tilde{E}\right| \ll\left|\omega_{e} \partial_{t} \tilde{E}\right|$ has been neglected. Equation (15) describes the evolution of the slowly varying amplitude $\tilde{E}$, as defined in (14).

We next proceed with the derivation of the equation for the low frequency part, $n_{l}$, of the departure from the equilibrium density $n_{0}$. After averaging over the fast time scale, we get a set of equations describing the low frequency part of the electron continuity equation, electron force equation and ion force equation,

$$
\begin{aligned}
& \frac{\partial n_{l}}{\partial t}+n_{0} \frac{\partial u_{l}}{\partial x}=0, \\
& \frac{\partial u_{l}}{\partial t}+\frac{e}{m_{e}} E_{l}+\frac{\kappa_{B} T_{e}}{n_{0} m_{e}} \frac{\partial n_{l}}{\partial x}-\frac{\hbar^{2}}{4 m_{e}^{2} n_{0}} \frac{\partial^{3} n_{l}}{\partial x^{3}}+\frac{e^{2}}{4 m_{e}^{2} \omega_{e}^{2}} \frac{\partial|\tilde{E}|^{2}}{\partial x}=0, \\
& \frac{\partial u_{l}}{\partial t}-\frac{e}{m_{i}} E_{l}=0 .
\end{aligned}
$$

Convective terms were disregarded in view of a weak Langmuir turbulence assumption, as detailed by Thornhill and ter Haar ${ }^{17}$ Eliminating $u_{l}$ and $E_{l}$ from Eqs. (16, 18) and assuming $m_{e} / m_{i} \ll 1$, we obtain

$$
\frac{\partial^{2} n_{l}}{\partial t^{2}}-c_{s}^{2} \frac{\partial^{2} n_{l}}{\partial x^{2}}+\frac{\hbar^{2}}{4 m_{i} m_{e}} \frac{\partial^{4} n_{l}}{\partial x^{4}}=\frac{\varepsilon_{0}}{4 m_{i}} \frac{\partial^{2}|\tilde{E}|^{2}}{\partial x^{2}}
$$

where $c_{s}=\left(\kappa_{B} T_{e} / m_{i}\right)^{1 / 2}$ is the ion-acoustic velocity. We call Eqs. (15) and (19) the quantum Zakharov equations.

For the following analysis, it is most convenient to normalize Eqs. (15) and (19). Normalized quantities are expressed as

$$
\begin{aligned}
& \bar{x}=2 \sqrt{\frac{m_{e}}{m_{i}}} \frac{x}{\lambda_{e}}, \quad \bar{t}=2 \frac{m_{e}}{m_{i}} \omega_{e} t, \\
& \bar{n}=\frac{1}{4} \frac{m_{i}}{m_{e}} \frac{n_{l}}{n_{0}}, \quad \bar{E}=\sqrt{\frac{\varepsilon_{0} m_{i}}{16 m_{e} n_{0} \kappa_{B} T_{e}}} \tilde{E},
\end{aligned}
$$

where $\lambda_{e}$ is the electron Debye length. In addition to (20, 21), we introduce the dimensionless quantum parameter

$$
H=\frac{\hbar \omega_{i}}{\kappa_{B} T_{e}}
$$

where $\omega_{i}=\left(n_{0} e^{2} / m_{i} \varepsilon_{0}\right)^{1 / 2}$ is the ion plasma frequency. The resulting system reads (we dropped bars for the sake of simplicity)

$$
\begin{aligned}
& i \frac{\partial E}{\partial t}+\frac{\partial^{2} E}{\partial x^{2}}-H^{2} \frac{\partial^{4} E}{\partial x^{4}}=n E \\
& \frac{\partial^{2} n}{\partial t^{2}}-\frac{\partial^{2} n}{\partial x^{2}}+H^{2} \frac{\partial^{4} n}{\partial x^{4}}=\frac{\partial^{2}|E|^{2}}{\partial x^{2}}
\end{aligned}
$$


The quantum parameter $H$ given in (22) expresses the ratio between the ion plasmon energy and the electron thermal energy. This is to be compared with the dimensionless parameter characterizing quantum effects in the two-stream quantum instability, $\frac{6}{\underline{6}}$ given by the ratio between electron plasmon and thermal energies. Here, the presence of ion-acoustic modes forces the appearance of ionic (inertia) parameters. Notice that for dense plasmas, $\underline{\underline{2}}$ with particle density about $10^{25}-10^{32} \mathrm{~m}^{-3}$ and temperature about $10^{5}-10^{7} \mathrm{~K}$, the parameter $H$ is not irrelevant at all. For a completely ionized hydrogen plasma in these ranges of densities and temperatures, $H$ typically goes from negligible values of order $10^{-5}$ up to values of order unity. The presence of large values of $H$ points to the possible experimental manifestation of quantum effects in the coupling between Langmuir and ion-acoustic modes in dense plasmas, particularly in astrophysical plasmas.

In the next section, the model (23) 244) is used to investigate two parametric instabilities extensively studied in classical plasmas: the decay instability and the four-wave interaction.

\section{PARAMETRIC INSTABILITIES}

\section{A. Decay instability}

Following strictly the treatment for the classical decay instability, $\frac{17}{p}$ consider the proposal

$$
\begin{aligned}
E & =E_{0} e^{i\left(k_{0} x-\omega_{0} t\right)}+E_{1}(t) e^{i\left(k_{1} x-\omega_{1} t\right)}, \\
n & =n_{1}(t) \cos (K x-\Omega t),
\end{aligned}
$$

now for the quantum Zakharov equations (23|24), where $E_{1}(t)$ and $n_{1}(t)$ are first-order quantities, and

$$
\begin{aligned}
& \omega_{0}=k_{0}^{2}+H^{2} k_{0}^{4}, \\
& \omega_{1}=k_{1}^{2}+H^{2} k_{1}^{4}, \\
& \Omega^{2}=K^{2}+H^{2} K^{4} .
\end{aligned}
$$

Notice that Eq. (29) is identical to the quantum dispersion relation obtained by Haas et al. $\underline{\underline{12}}$ (Section $\mathrm{V}$ ) except for the value of $H$ which, due to the use of a quantum equation of state, has a different definition there.

Also, there are the usual matching conditions

$$
k_{0}=k_{1}+K, \quad \omega_{0}=\omega_{1}+\Omega,
$$


corresponding to momentum and energy conservation respectively. These conditions describe the decay of one quantum Langmuir wave, with dispersion relation (27), into other quantum Langmuir wave, with dispersion relation (28), and a quantum ion-acoustic wave, with dispersion relation (29).

Linearizing the quantum Zakharov equations, we obtain

$$
\begin{aligned}
& i \dot{E}_{1} e_{1}=\frac{E_{0} n_{1}}{2}\left(e_{+}+e_{-}\right) e_{0}, \\
& \left(\frac{\ddot{n_{1}}}{2}-i \Omega \dot{n_{1}}+K^{2} E_{0} E_{1}^{*}\right) e_{+}+\left(\frac{\ddot{n_{1}}}{2}+i \Omega \dot{n}_{1}+K^{2} E_{0}^{*} E_{1}\right) e_{-}=0,
\end{aligned}
$$

where use has been made of the notation

$$
e_{0,1}=\exp \left[i\left(k_{0,1} x-\omega_{0,1} t\right)\right], \quad e_{ \pm}=\exp [ \pm i(K x-\Omega t)]
$$

The resonant part [satisfying (30)] of (31) gives

$$
n_{1}=\frac{2 i}{E_{0}} \dot{E}_{1}
$$

while (32) gives

$$
\frac{\ddot{n}_{1}}{2}+i \Omega \dot{n_{1}}+K^{2} E_{0}^{*} E_{1}=0 .
$$

For $\dot{E}_{1}=i \omega E_{1}$, elimination of $n_{1}$ leaves us with

$$
\omega^{3}+2 \Omega \omega^{2}+K^{2}\left|E_{0}\right|^{2}=0,
$$

which is formally identical to the dispersion relation for the classical decay instability. Hence, all conclusions valid for the classical case can be immediately extended to the quantum case. In particular, for $\Omega \gg|\omega|$, so that the cubic term can be neglected in (36), and for $\omega=i \gamma$, we obtain the growth rate

$$
\gamma=\frac{K\left|E_{0}\right|}{\sqrt{2 \Omega}}
$$

In all situations, the discriminant ${ }^{20}$ of the cubic equation (36) is positive and there are one real and two complex conjugate solutions for this equation, one of which is necessarily unstable.

To conclude, there is a formal similarity between the classical and quantum decay instabilities. The only differences remain in the dispersion relations (27) 29), for the quantum Langmuir and ion-acoustic modes. The quantum dispersion relations, however, produces a 
saturation effect not present in the classical case (see Fig. 1). Combining (29) and (37), we obtain

$$
\gamma=\frac{\sqrt{K}\left|E_{0}\right|}{\sqrt{2}\left(1+H^{2} K^{2}\right)^{1 / 4}},
$$

for which a maximum value $\gamma_{\max }=\left|E_{0}\right| / \sqrt{2 H}$ is obtained for $K \rightarrow \infty$. This is to be compared with the classical case $(H=0)$ where $\gamma$ grows with no bound as $K$ increases. Even if the quantum effects do not imply stabilization, they limit the instability to a fixed maximum growth rate.

For dense astrophysical hydrogen plasmas, ${ }^{2}$ where $n_{0} \sim 10^{32} \mathrm{~m}^{-3}$ and $T_{e} \sim 10^{5} \mathrm{~K}$, we

obtain $H \sim 1$ and $\gamma_{\max }=\left|E_{0}\right| / \sqrt{2}$. For laser hydrogen plasmas, however, to the best of our knowledge, such high densities are not yet attainable. For today's typical values ${ }^{3}$ of $n_{0} \sim 10^{28} \mathrm{~m}^{-3}$ and $T \sim 10^{5} \mathrm{~K}$, we obtain a modest value of $H \sim 10^{-2}$. The smallness of the quantum effects for modulational instabilities in laser plasmas follows from the fact that, in this respect, the relevant parameter $H$ is defined as the ratio of the ion plasmon energy to the electron thermal energy. If the pertinent quantum parameter were the ratio between the electron plasmon energy and the electron thermal energy, as in the quantum two-stream instability, ${ }^{6}$ the lower mass of the electrons would increase significantly $H$. For laser plasmas with the same typical values as before, we would have $H \sim 0.4$. Of course, we are not saying that quantum effects are irrelevant for laser plasmas: they can show up when the Landau length becomes comparable to the de Broglie wavelength, in which case $\hbar \omega_{c} \sim \kappa_{B} T_{e}$, where $\omega_{c}$ is the cyclotron frequency associated with the laser field, or when the electrons are degenerated. $\stackrel{3}{ }^{-}$

\section{B. Four-wave instability}

The general instability involving the interaction between one single finite-amplitude Langmuir wave, two other Langmuir waves and one ion-acoustic wave can be obtained by choosing 18

$$
\begin{aligned}
E(x, t) & =E_{0} \exp \left(-i \omega_{0} t+i k_{0} x\right)+E_{+} \exp \left[-i\left(\omega_{0}+\omega\right) t+i\left(k_{0}+k\right) x\right] \\
& +E_{-} \exp \left[-i\left(\omega_{0}-\omega^{*}\right) t+i\left(k_{0}-k\right) x\right] \\
n(x, t) & =\tilde{n} \exp (-i \omega t+i k x)+\text { c.c }
\end{aligned}
$$


where the amplitudes $E_{+}, E_{-}$and $\tilde{n}$ are all first order quantities. We choose the equilibrium solution $E(x, t)=E_{0} \exp \left(-i \omega_{0} t+i k_{0} x\right), n(x, t)=0$ to satisfy Eqs. (23,24) with $E_{0}$ real. This implies the relation

$$
\omega_{0}=k_{0}^{2}+H^{2} k_{0}^{4}
$$

The last equation can be found also from the dispersion relation for quantum Langmuir modes [equation (8) ] taking $\omega_{e}=0$ and the recalling of variables. In fact, $\omega_{e}$ is absorbed in the electric field through (14). In conclusion, (41) shows a quantum Langmuir mode.

The forms (39,40) when inserted in Eq. (23) yield

$$
\begin{aligned}
& \left(\omega_{0}+\omega\right) E_{+}-\left(k_{0}+k\right)^{2} E_{+}=\tilde{n} E_{0}+H^{2}\left(k_{0}+k\right)^{4} E_{+}, \\
& \left(\omega_{0}-\omega^{*}\right) E_{-}-\left(k_{0}-k\right)^{2} E_{-}=\tilde{n}^{*} E_{0}+H^{2}\left(k_{0}-k\right)^{4} E_{-} .
\end{aligned}
$$

When combined, Eqs. (24), (41,42) and the complex conjugate of (43) give the following dispersion relation:

$$
D_{s} D_{1} D_{2}=k^{2} E_{0}^{2}\left(D_{1}+D_{2}\right)
$$

in which

$$
\begin{aligned}
& D_{s}=\omega^{2}-k^{2}-H^{2} k^{4}, \\
& D_{1}=\omega-k^{2}-2 k_{0} k-H^{2}\left(k^{4}+4 k_{0} k^{3}+6 k_{0}^{2} k^{2}+4 k_{0}^{3} k\right), \\
& D_{2}=-\omega-k^{2}+2 k_{0} k-H^{2}\left(k^{4}-4 k_{0} k^{3}+6 k_{0}^{2} k^{2}-4 k_{0}^{3} k\right) .
\end{aligned}
$$

Notice that the limit $H \rightarrow 0$ recovers the classical dispersion relation for the four-wave interaction. ${ }^{18}$

The dispersion relation (44) is a fourth order polynomial in $\omega$ that, in general, can only be analyzed numerically. However, the simplest case $\omega_{0}=k_{0}=0$ can be investigated analytically. For a purely growing instability $\omega=i \gamma$ the dispersion relation (44) becomes

$$
\left[\gamma^{2}+k^{2}+H^{2} k^{4}\right]\left[\gamma^{2}+\left(k^{2}+H^{2} k^{4}\right)^{2}\right]=2 k^{2} E_{0}^{2}\left(k^{2}+H^{2} k^{4}\right)
$$

Solving for $\gamma^{2}$, there follows two roots,

$$
\begin{aligned}
\gamma^{2} & =-\frac{1}{2}\left(k^{2}+H^{2} k^{4}\right)\left(1+k^{2}+H^{2} k^{4}\right) \\
& \pm \frac{1}{2}\left(k^{2}+H^{2} k^{4}\right)^{1 / 2}\left[\left(k^{2}+H^{2} k^{4}\right)\left(1-k^{2}-H^{2} k^{4}\right)^{2}+8 k^{2} E_{0}^{2}\right]^{1 / 2}
\end{aligned}
$$


one of them necessarily negative (stable mode). The other root is positive (unstable mode) provided

$$
E_{0}^{2}>\frac{k^{2}}{2}\left(1+H^{2} k^{2}\right)^{2}
$$

This recovers the classical instability condition for the four-wave interaction ${ }^{18}$ when $H \rightarrow 0$. However, there is a new, quantum effect of instability suppression provided

$$
H^{2} \geq \frac{\sqrt{2} E_{0}-k}{k^{3}}
$$

This is in agreement with the overall stabilization that quantum diffraction effects produce in high density plasmas. ${ }^{6.7}$ In fact, for sufficiently large $H$ there is no transfer of energy from the original quantum Langmuir mode to the two new quantum Langmuir modes and to the quantum ion-acoustic mode.

Let us consider in more detail the potentially unstable mode described by the positive root in (49). In Fig. 2, we show $\gamma^{2}$ versus $k^{2}$ for $H=0, H=0.5$ and $H=0.9$, with $E_{0}=0.5$. The instability region $\left(\gamma^{2}>0\right)$ in $k$-space becomes narrower for bigger $H$. Also, the maximum $\gamma^{2}$ becomes smaller the larger the quantum effects. This latter result is analytically supported by an expansion of the positive root of Eq. (49). The wavenumber for maximum growth rate, $k_{\max }$ can be calculated, in a $O\left(k^{5}\right)$ approximation, by expanding Eq. (49) up to fifth order in $k$. This perturbation analysis is interesting since $d \gamma / d k=0$ is not soluble in closed form if we use the exact expression for (49). The result of the expansion procedure is shown in Fig. 3, where the wavenumber $k_{\max }$ for maximum growth rate when $E_{0}=0.5$ is shown as a function of $H$. (Notice the extended domain of the function, beyond the reasonable limit of $H \cong 1$.) Using this $k_{\max }$ we obtain a somewhat complicated expression which can be used to calculate the associated growth rate $\gamma$. Using a computer algebra program, we can easily obtain $\gamma_{\max }=E_{0}^{2}\left(1-E_{0}^{2}-0.87 H^{2}\right)$, a result valid up to $O\left(E_{0}^{4}\right)$. This is an approximate equation showing that quantum effects produce stabilization. The approximations adopted are justified in view of our assumptions of long wavelengths and weak turbulence (small electric field amplitudes). For dense astrophysical plasmas with $H \sim 1$, as in the decay instability case, we would get $\gamma_{\max }=E_{0}^{2}\left(0.13-E_{0}^{2}\right)$, a significant difference in comparison with the classical case where $\gamma_{\max }=E_{0}^{2}\left(1-E_{0}^{2}\right)$.

In order to further assess the role of quantum effects in the four-wave interaction process, we performed a numerical study of (44) for general $k_{0} \neq 0$. Figure 4 displays the real (solid lines) and imaginary (dashed lines) parts of $\omega$ as a function of $k$. Both uncoupled 
(i.e., $E_{0} \approx 0$ ) and coupled cases are considered, for three different values of $H$. Due to the symmetry $(k, \omega) \leftrightarrow(-k,-\omega) \Rightarrow D_{1} \leftrightarrow D_{2}$, of the dispersion relation (44), we consider only positive values of the wavenumber, around the overlay region of the branches $D_{s}$ and $D_{2}$, where instability occurs. In the uncoupled case, $k=2 k_{0}$ is a root of $D_{2}$ when $\omega=0$, for both classical and quantum cases. Also, the plots of $D_{s}$ and $D_{2}$ branches touch each other at isolated points while, when $E_{0} \neq 0$, overlay occurs for a whole finite interval of $k$, signalizing wave instability. The first column of plots shows that, for a fixed $k_{0}$, both uncoupled curves raise with $H$, implying reduction of the interval in $k$ where instability settles down. This can be checked against the corresponding figures in the second and third columns, where a contraction of the unstable interval is clearly seen.

Denote the unstable interval in $k$ by $I_{k}=\left(k_{a}, k_{b}\right)$. For higher pump energy $E_{0}$, the third column of Fig. 4 shows an overall contraction of $I_{k}$. This results from the gradual shift of $k_{a}$ to the right and $k_{b}$ to the left, due to the quantum effects. For the relevant range of values $0 \leq H \leq 1$, less severe attenuations occur for the maximum growth rate, compared to those found for the unstable interval in $k$. Thus, the numerical results show that the quantum effect inhibits the spreading of energy among different modes. In fact, assume that for a specific $k, N_{I}=\left(k_{b}-k_{a}\right) / k$ represents a first estimation for the number of active modes at the beginning of the process. Then, the contraction of $I_{k}$ implies that the Langmuir fluctuations in quantum plasmas might represent more coherent configurations, i.e., having less effective modes when compared to the corresponding classical situation, an issue to be checked by a direct numerical simulation and, possibly, by an experiment.

\section{NONLINEAR ANALYSIS AND OPEN QUESTIONS}

An important regime of the classical Zakharov equations concerns its static limit. In this case, the classical Zakharov system do possess soliton solutions described by a nonlinear Schödinger equation. ${ }^{17}$ The procedure for the static limit of the quantum Zakharov equations considers the approximation $\partial^{2} n / \partial t^{2} \approx 0$ in (24). This gives immediately

$$
n=-|E|^{2}+H^{2} \frac{\partial^{2} n}{\partial x^{2}}
$$

Equation (52), inserted in Eq. (23), yields

$$
i \frac{\partial E}{\partial t}+\frac{\partial^{2} E}{\partial x^{2}}+|E|^{2} E=H^{2}\left(\frac{\partial^{4} E}{\partial x^{4}}+E \frac{\partial^{2} n}{\partial x^{2}}\right) .
$$


In the classical limit $H \rightarrow 0$, the right-hand side of Eq. (53) vanishes and we recover the nonlinear Schrödinger equation with its soliton solutions. In the quantum case, however, equations (52 53) form a coupled, nonlinear system. We have not been able to find localized, analytical solutions for this system. In fact, the usual reduction procedure of searching for solutions in the form

$$
E=F(x-M t) \exp (i[k(x-u t)+\delta]), \quad n=G(x-M t),
$$

for real $F, G, k, M, u$ and $\delta$ produces a complicated fourth-order system of coupled, nonlinear equations. The existence of soliton solutions for this system remains an open question. It seems that a numerical analysis could help in this respect but we believe that this issue should be more appropriately treated in a future work.

Another avenue in nonlinear studies of the quantum Zakharov equation concerns its simultaneous semiclassical and static limit. Substituting (152) into (53) and retaining only terms up to $O\left(H^{2}\right)$ produces the decoupled equation

$$
i \frac{\partial E}{\partial t}+\frac{\partial^{2} E}{\partial x^{2}}+|E|^{2} E=H^{2}\left(\frac{\partial^{4} E}{\partial x^{4}}-E \frac{\partial^{2}|E|^{2}}{\partial x^{2}}\right) .
$$

Equation (55) can be used to study perturbations of the classical NLS soliton solutions. The terms proportional to $H^{2}$, in Eq. (55), will probably modify the dispersion-nonlinearity equilibrium, which is the ultimate responsible for the soliton existence.

More formal aspects of the Zakharov equations have to do with its variational formulation and the associated Noether currents. ${ }^{21}$ In particular the quantum Zakharov equations preserve the number of plasmons $\int|E|^{2} d x$ of the high frequency electric field, as a consequence of the associated conservation law

$$
\frac{\partial \rho}{\partial t}+\frac{\partial J}{\partial x}=0
$$

where $E(x, t)=A(x, t) \exp (i \theta(x, t))$, with $A=A(x, t)$ and $\theta=\theta(x, t)$ real amplitude and phase functions, and

$$
\begin{aligned}
\rho & =A^{2} \\
J & =2 A^{2} \frac{\partial \theta}{\partial x}-2 H^{2}\left[A^{2} \frac{\partial^{3} \theta}{\partial x^{3}}+2 A \frac{\partial A}{\partial x} \frac{\partial^{2} \theta}{\partial x^{2}}\right. \\
& \left.-2 A^{2}\left(\frac{\partial \theta}{\partial x}\right)^{3}-2\left(\frac{\partial A}{\partial x}\right)^{2} \frac{\partial \theta}{\partial x}+4 A \frac{\partial^{2} A}{\partial x^{2}} \frac{\partial \theta}{\partial x}\right] .
\end{aligned}
$$


Notice the extra contribution proportional to $H^{2}$ to the plasmons current.The conservation law (56) comes from the imaginary part of (23) and hence contains no contribution from $n$. A proper formulation of the remaining conservation laws (momentum and energy) of the system is an open question to be tackled, preferably in accordance with symmetry principles of an associated action functional. Other important issues concern the search for coherent solutions of the quantum Zakharov equations, namely quantum solitons and quantum cavitons.

Still another issue related to the nonlinear analysis of Eqs. (23,24) concerns thermalization and recurrence. For periodic boundary conditions, the classical NLS does not exhibit thermalization and, therefore, is generically recurrent.22.23 The classical procedure to address such questions is based on estimations for the number of active modes $N_{A}$, from the Rayleigh quotient. An upper bound estimation for this number is provided by two invariants: the number of plasmons and a momentum-like invariant which, in our case, is not yet known. For classical regimes, numerical simulations show that the conclusions can, in general, be extended to the non-integrable Zakharov system, when considered as a perturbation of the NLS regime. ${ }^{24}$ In fact, it has been shown that, at least for some period of time, the constancy of the momentum-like quantity is approximately satisfied. Moreover, numerical simulations show that the elementary estimation presented in the last section, i.e., $N_{A} \approx N_{I}$, can yield quite good results when applied to the full Zakharov equations. Under this viewpoint, the contraction of the $k$-unstable interval due to $H \neq 0$, verified in subsection (IVB), suggests that the distribution of energy is less intense in quantum plasmas when compared with the classical case. Therefore, quantum effect would favor recurrence in Langmuir modulational regimes.

To finalize, we can derive some exact solutions for the quantum Zakharov equations (23) 24) if we consider pure ion-sound waves obtained by taking $E=0$. With zero electric field, the density perturbation satisfies the undriven equation

$$
\frac{\partial^{2} n}{\partial t^{2}}-\frac{\partial^{2} n}{\partial x^{2}}+H^{2} \frac{\partial^{4} n}{\partial x^{4}}=0
$$

This linear fourth-order evolution equation was investigated using the method of Lie symmetries $^{25}$ and we found time and space translation symmetries, as well as a scale symmetry resulting from the linearity. The $H^{2}$ term breaks down the Lorentz invariance endowed by the classical model for pure ion-sound waves, so that arbitrary waves travelling at the 
ion-sound velocity can not be constructed. Nevertheless, exact solutions for Eq. (59) can be found supposing $n=\bar{n}(x-c t)$, for constant $c$ and for $\bar{n}$ a function to be determined. For

$c^{2}>1$, corresponding to supersonic flow, and disregarding an integration constant associated to non-bound solutions, we get periodic solutions of the form

$$
n=a+b \cos \left(\frac{\sqrt{c^{2}-1}}{H}(x-c t)+\delta\right),
$$

where $a, b$ and $\delta$ are numerical constants. This similarity solution is an arbitrary amplitude solution. Notice that quantum effects increase the spatial frequency of oscillations in the reference frame of the travelling wave.

\section{CONCLUSION}

We obtained a general model to analyze the coupling between Langmuir waves and ionacoustic waves, in a quantum setting. The model was shown to be appropriate to the four-wave interaction and quantum effects have been shown to provide stabilization of a classically unstable mode. In the case of the decay instability, a formal similarity with the classical case is identified, except for small differences in the dispersion relations, representing quantum corrections. We also identified a dimensionless quantum parameter given by the ratio of the ion plasmon and electron thermal energies. As pointed out before, this quantum parameter may not be small, at least for dense plasmas.

The consequences of our results on todays laboratory or technological plasmas are not yet fully assessed since, for present conditions, $H \ll 1$ in these applications. However quantum effects may imply important consequences in the behavior of high density astrophysical plasmas, where $H \sim 1$ is easily found. In this case, as we pointed out, quantum effects cause an overall reduction in the wave-wave interaction level. Specifically and in contrast to the classical case, the decay instability growth rate is bounded for large wavenumbers. Growth rate reduction also occurs for the four-wave interaction. Besides, suppression is also verified in the length of the unstable spectral range, implying spectral focusing, i.e., a restriction on the range of possible unstable wave-numbers. This focusing effect may extend to quite long periods of time, indicating that the recurrence properties verified in the classical Zakharov equation are enhanced by the quantum effects.

A number of open questions remains to be addressed. Of course, a complete analysis of the 
linear dispersion relation of the quantum Zakharov system have to be done. This may require a full three-dimensional treatment, with the inclusion of electromagnetic coupling between Langmuir and ion-acoustic modes. An additional important point are the nonlinear effects, some of them briefly discussed in Section V, which may deserve a more careful scrutiny. To conclude, the huge amount of physical and mathematical aspects already assessed in the classical Zakharov equations certainly have quantum counterparts which ask for an equally careful investigation.

\section{Acknowledgments}

This work was partially supported by Conselho Nacional de Desenvolvimento Científico e Tecnológico - CNPq. One of us (L. G. G.) gratefully acknowledges Universidade do Vale do Rio dos Sinos - UNISINOS for hospitality and support during the preparation of this work. 
1 P. A. Markowich, C. A. Ringhofer and C. Schmeiser, Semiconductor Equations (Springer, Vienna, 1990).

2 Y. D. Jung, Phys. Plasmas 8, 3842 (2001); M. Opher, L. O. Silva, D. E. Dauger, V. K. Decyk and J. M. Dawson, Phys. of Plasmas 8, 2454 (2001).

3 D. Kremp, Th. Bornath, M. Bonitz and M. Schlanges, Phys. Rev. E 60, 4725 (1999); Th. Bornath, M. Schlanges, P. Hilse and D. Kremp, Phys. Rev. E 64, 026414 (2001).

4 G. Manfredi and M. R. Feix, Phys. Rev. E 53, 6460 (1996).

5 S. Mola, G. Manfredi and M. R. Feix, J. Plasma Phys. 50, 145 (1993).

6 F. Haas, G. Manfredi and M. R. Feix, Phys. Rev. E 62, 2763 (2000); D. Anderson, B. Hall, M. Lisak and M. Marklund, Phys. Rev. E 65, 046417 (2002); F. Haas, G. Manfredi and J. Goedert, Phys. Rev. E 64, 26413 (2001); F. Haas, G. Manfredi and J. Goedert, Braz. J. Phys. 33, 128 (2003).

7 N. Suh, M. R. Feix and P. Bertrand, J. Comput. Phys. 94, 403 (1991).

8 R. Fedele, P. K. Shukla, M. Onorato, D. Anderson and M. Lisak, Phys. Lett. A 303, 61 (2002).

9 P. Bertrand, N. van Tuan, M. Gros, B. Izrar, M. R. Feix and J. Gutierrez, J. Plasma Phys. 23, $401(1980)$.

10 C. Gardner, SIAM J. Appl. Math. 54, 409 (1994); M. G. Ancona and G. J. Iafrate, Phys. Rev. B 39, 9536 (1989); M. V. Kuzelev and A. A. Rukhadze, Physics Uspekhi 42, 687 (1999).

11 G. Manfredi and F. Haas, Phys. Rev. B 64, 075316 (2001).

12 F. Haas, L. G. Garcia, J. Goedert and G. Manfredi, Phys. Plasmas 10, 3858 (2003).

13 Z. Chen, B. Cockburn, C. Gardner and J. Jerome, J. Comp. Phys. 117, 274 (1995); C. Gardner and C. Ringhofer, SIAM J. Appl. Math. 58, 780 (1998).

14 J. R. Zhou and D. K. Ferry, IEEE Trans. Electron. Dev. 40, 421 (1993).

15 J. L. López, Phys. Rev. E 69, 026110 (2004).

16 V. E. Zakharov, Zh. Eksp. Teo. Fiz 62,1745 (1972) [Sov. Phys. JETP 35, 908 (1972)].

17 S. G. Thornhill and D. ter Haar, Phys. Rep. 43, 43 (1978).

18 D. R. Nicholson, Introduction to Plasma Theory (Wiley, New York, 1983).

19 Yu. L. Klimontovich and V. P. Silin, Zh. Eksp. Teor. Fiz. 23, 151 (1952); J. E. Drummond, Plasma Physics (McGraw-Hill, New York, 1961); N. Maafa, Physica Scripta 48, 351 (1993). 
20 I. S. Gradshteyn and I. M. Rhyzik, Tables of Integrals, Series and Products (Academic Press, New York, 1965).

21 J. Gibbons, S. G. Thornhill, M. J. Wardrop and D. H. ter Haar, J. Plasma Phys. 17, 153 (1977).

22 A. Thyagaraja, Phys. Fluids, 22, 11 (1979).

23 A. Thyagaraja, Phys. Fluids, 24, 11 (1981).

24 L. P. L. de Oliveira, F. B. Rizzato and A. C. -L. Chian, J. Plasma Physics, 58, 441 (1997).

25 P. J. Olver, Applications of Lie Groups to Differential Equations, Graduate Texts in Mathematics no. 107. (Springer-Verlag, New York, 1986). 


\section{FIGURE CAPTIONS}

FIG. 1. Growth rate of the decay instability for $E_{0}=0.5$ and $0 \leq H \leq 1.5$, as indicated. Notice the quick saturation effect for $H>0$.

FIG. 2. $\gamma^{2}$ as a function of $k^{2}$ for the positive root in the dispersion relation (49) for the four-wave instability. We have $E_{0}=0.5, H=0$ (full line), $H=0.5$ (dashed line) and $H=0.9$ (dotted line).

FIG. 3. Wave-number $k_{\max }$ for maximum growth rate of the four-wave instability, as a function of $H$, calculated to $O\left(k^{5}\right)$ and $E_{0}=0.5$.

FIG. 4. Real (solid lines) and imaginary (dashed lines) components of the frequency $\omega$ as a function of $k$ for uncoupled (frames a1, a2 and a3) and coupled cases (frames b1 to c3). From top to bottom, $H=0, H=0.5$ and $H=0.9$, respectively. From left to right, $E_{0}=0$, $E_{0}=0.5$ and $E_{0}=0.5$. For the first and second columns $k_{0}=0.5$; for the third column,

$k_{0}=0.75$. In the first frame, $D_{i}(i=s, 1,2)$ indicate the various branches of (44). A similar labelling applies to all the frames. 